\title{
Characterization of the Noise in Secondary Ion Mass Spectrometry Depth Profiles
}

\author{
D. P. Chu, M. G. Dowsett, and G. A. Cooke \\ Department of Physics, University of Warwick, Coventry CV4 7AL, UK
}

The noise in the depth profiles of secondary ion mass spectrometry (SIMS) is studied using different samples under various experimental conditions. Despite the noise contributions from various parts of the dynamic SIMS process, its overall character agrees very well with the Poissonian rather than the Gaussian distribution in all circumstances. The Poissonian relation between the measured mean-square error (MSE) and mean can be used to describe our data in the range of four orders. The departure from this relation at high counts is analyzed and found to be due to the saturation of the channeltron used. Once saturated, the detector was found to exhibit hysteresis between rising and falling input flux and output counts.

PACS numbers: 82.80.Ms, 85.40.Qx, 07.50.Hp 


\section{INTRODUCTION}

Secondary ion mass spectrometry (SIMS) is widely used for the measurement of doping, impurity and matrix profiles in semiconductors. In this application, the concentration data required may span 10 orders of magnitude overall, and 4-6 orders for a particular species. As the SIMS data do not directly represent the true element profile, a data quantification procedure is necessary. Various methods from simple linear mapping (ion dose to depth, signal to concentration) to deconvolution using response functions of various types [1]. Due to the complexity of the dynamic SIMS profile process and the large record dynamic range required, a very careful and unbiased treatment of the measured SIMS data is vital to the success of a deconvolution method to be used. It is widely accepted that one of the best

deconvolution methods in use is based on maximum entropy [2]. We have developed and applied a maximum entropy method to the deconvolution of SIMS profiles [3, [4]. However, the use of the correct noise model is essential in high dynamic range deconvolution, since the adoption of arbitrary noise models can lead to quite different results - both in the depth resolution of the recoved profile, their dynamic range, and the behaviour in the low counts regime. Therefore, the accurate determination of the noise characteristics is an indispensable part of the self-consistent use of deconvolution methods.

\section{NOISE IN SIMS DATA}

Noise in SIMS data can be attributed to four main sources: (1) the change of the primary ion flux due to statistical fluctuations in the ion source; (2) the fluctuation in the effective secondary ion yield due to the statistical nature of sputtering; (3) the statistics 
of the secondary ion detection process, probably dominated by ion to electron conversion probabilities at the first collision with the detector (in the case of this study, a Gallileo Corp. model 4816 channeltron); (4) there may also be additional effects due to the locally random distribution of atoms of the analytic species, significant when small volumes are analysed. For most depth profiles, integrating the signal over the volume of at least $10^{9} \mathrm{~nm}^{3}$ per data point makes this last contribution insignificant. In general, it is very difficult to distinguish the noise generated by different sources within the same process. The data we normally collect and quantify in dynamic SIMS are the counts for a pixel or frame, where the latter is effectively the sum over a set of the pixels at a certain depth, in a period of time during which the operating conditions of the instrument are assumed constant. In other words, we can only study the overall SIMS noise in our data.

Separating noise from signal is not easy and convincing if the signal itself is changing in time. It is better to keep the signal as steady as possible, i.e. to maintain the mean value of signal unchanged during a period of time, and then deduce the noise from the collected data. To obtain a steady signal in dynamic SIMS profiling, we may use two different approaches. The first one is to study so-called staircase samples which are made of different levels of constant doping of a specific element in different ranges of depth. Various levels of stable signals in the corresponding mass channel will be obtained when we profile through the samples. The other approach is to collect data from a certain mass channel which corresponds to a uniform distribution of an element while we change the primary ion current from time to time. Different currents will generate different fluxes of secondary ions varying about a constant mean flux.

A thorough description of a noise requires its frequency distribution [5]. If it differs from 
that of the noise model we wish to use, the accuracy of the deconvolution process will be compromised. However, there is no real problem in which we have sufficient information to fully describe the frequency spectrum [6]. Fortunately, we do not need so much information about the noise in our quantification procedure. Usually the relation between the magnitude of a noise, which is characterized by its mean-square error (MSE), $\sigma^{2}=\sum_{i}\left(s_{i}-s_{m}\right)^{2} / N$ where $s_{i}$ is the signal of the $i$-th frame out of a total of $N$ frames, and the corresponding mean, $s_{m}=\sum_{i} s_{i} / N$, will be sufficient in most cases.

We would like to stress here that the relation between the MSEs and mean values is not a full description of the noise, but rather a description of our state of knowledge about the noise. It is related to the fact that we want to be fairly sure that we choose a set of possible solutions big enough to include the true solution during our deconvolution process [6]. Choosing too "narrow" a noise distribution will result in data containing structure for which there is no evidence.

\section{CHARACTERIZATION OF THE NOISE}

The samples used in this study were four boron staircase structures in silicon grown by MBE at temperatures between 600 and $760{ }^{\circ} \mathrm{C}$. Doping levels varied from $3 \times 10^{16}$ to $1 \times$ $10^{19}$ atoms $/ \mathrm{cm}^{3}$. The samples are designated $14.01,17.03,25.01$ and 39.02 in what follows. Depth profiles were obtained on our EVA 3000 instrument using normally incident $\mathrm{O}_{2}^{+}$ions at beam energies of either $500 \mathrm{eV}$ or $2 \mathrm{keV}$ (typical of high depth resolution conditions) using 600 and $500 \mu \mathrm{m}$ square craters with $10 \%$ areal gates (applied digitally, post acquisition). A typical boron profile from sample 39.02 is shown in Fig. 1. Two types of study were carried out: (i) profiles of the boron staircase and silicon matrix intensity at fixed beam current, 
and (ii) profiles of the silicon matrix channel at a variety of beam currents and sample bias, $V_{s}$, to vary the input flux to the channeltron over a large dynamic range. In this way it was possible to establish a relationship between $\sigma$ and $s_{m}$ over a wide range of samples and

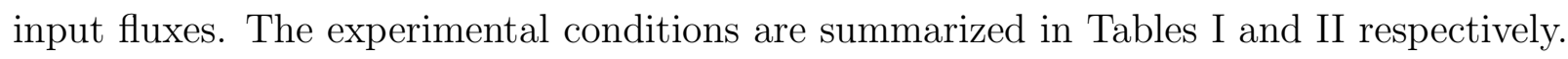

The $\sigma$ and $s_{m}$ of the noise calculated from the profiles of the staircase samples study (i) are plotted in Fig. 2, where the $s_{m}$ were obtained from the segments of data in which the concentrations are supposed to be constant. In Fig. 2, we can see that all the $\sigma$ and $s_{m}$ agree well with the Poissonian relation $\sigma=s_{m}^{1 / 2}$ rather than the Gaussian one $\sigma=\left(s_{m} / 2\right)^{1 / 2}$ for $s_{m} \leq 10^{4}$. The data start to depart from this relation gradually when the $s_{m}$ is larger. Since the boron concentrations in our staircase samples were low, we have the data points corresponding to the respective channels in two spatially separated areas on the plot.

Study (ii) determines whether or not the silicon has the same noise model as the boron, despite the apparent departure from the Poissonian behaviour shown in Fig. 2 .

The corresponding $\sigma$ and $s_{m}$ are shown in Fig. 3. Again we can see the Poissonian model suits our data well for $s_{m} \leq 10^{5}$ with a departure for for larger input fluxes to the channeltron. We conclude therefore that under our experimental conditions the major noise source or the combination of noise sources is effectively Poissonian.

The MSEs and means were calculated from data of finite length. We chose long segments with large enough number of frames so that the statistical errors involved are much smaller than the quantities in which we are interested. Some of the data exhibited a long term drift of a few $\%$ in intensity due to the normal behaviour of a plasma ion source. To prevent excessive MSE values from such data, MSE's were calculated from long segments of data where each short segments had almost equal means with no obvious systematic behaviour. 
Some long segments consisted of a linear drift on the mean, which was recognized by the linear relation of the corresponding means for each short segment and the almost the same magnitude of MSEs after subtracting a liner drift background. In this situation, the overall means were assigned to such MSEs. This may lead to a small fraction of error into the relation of the MSE and mean, but the relative error involved is no more than the maximum relative difference of the means of short segments within a long segment, which is negligible in our calculations.

To make sure that the data used in our calculations only involve random noise, we used two methods to analyse them: time-frequency technique [0] and random walk method [8]. The first method uses Wigner distribution $W(i, \omega)=(1 / 2 \pi) \int s^{*}(i-t / 2) s(i+$ $t / 2) \exp (-i \omega t) d t$ and is capable of revealing any ordering of frequency components in time, and the second method calculates the root mean square fluctuation of the displacement $F(j)=\left(<\Delta S(j)^{2}>-<\Delta S(j)>^{2}\right)^{1 / 2}$ where $\Delta S(j)=S(j)-S(1)$ and the running sum $S(j)=\sum_{i=1}^{j} s_{i}$ and can detect the presence of long-range correlations existing in data. No pattern with order was found in our data and the $F(j) \sim j^{\alpha}$ gives $\alpha \rightarrow 1 / 2$ for large $j$ as expected in uncorrelated time series.

\section{HYSTERESIS EFFECT OF THE DETECTOR}

Note that the MSE of noise in Figs. 2 and 3 becomes graduately larger than what the Poissonian relation predicates as the counts $>10^{4}-10^{5}$. It appears only to be related to the input flux to the detector. For a better understanding of this phenomenon, we measured the counts per frame in the silicon channel as a function of the primary beam current, $I_{p}$. See Figs. [(a) and (b). We found that the counts appear, as expected, to be proportional to the 
$I_{p}$ with approximately the same slope when they are $<10^{4}$ per second. When the output of the channeltron $>10^{4}$ counts per second, the channeltron starts to saturate. When the $I_{p}$ is increased further, the output drops as the pulse amplitude falls below the counting threshold. We therefore attribute the departures of the noise from the Poissonian relation in Figs. 2 2 and 3 to the channeltron saturation. A simple analysis shows that the MSE of counts saturates slower than the corresponding mean as the channeltron approaches its saturation. This agrees qualitatively with the departure trends in both Figs. 2 and 3 .

A hysteresis effect on the channeltron output was found as we raised the $I_{p}$ to saturate the channeltron and then reduced it to the normal value. We see in Fig. 国(b) that although the slopes for the linear regimes before and after the channeltron is saturated are nearly the same, the later output is about one order lower than the previous one for the same input flux. This may possibly be explained by degredation of the surface of the channeltron capillary as a result of the large number of secondary electrons flowing at the anode. This effect would make it very difficult to quantify SIMS data if the channeltron is saturated at some stages of profiling.

\section{DISCUSSION AND CONCLUSION}

The Poissonian distribution is a result of "law of small probability" given by Poisson. In SIMS, the ion count is much smaller than the number of secondary ions ejected. This ratio, known as the useful yield, is $\sim 10^{-6}-10^{-2}$ for $\mathrm{B}$ and Si respectively on various instruments [9:10]. The ratio of counted ions to input primary ions is likely to be orders of magnitude

smaller still. Such a small probability will normally lead to a statistics of Poissonian type, and several Poissonian stochastic processes combined together are still Poissonian. 
We conclude that the noise in our dynamic SIMS depth profiles is universally Poissonian despite of the change of samples and experimental conditions. Consequently, its MSE relates to the mean accurately as described by the Poissonian distribution in all the linear dynamic range of our experiments. The departure from this relation is due to the saturation effect of the channeltron used.

It is worth mentioning that noise characterization is unconsciously or deliberately ignored in many situations. In fact, when one uses a certain procedure of noise data processing, a particular distribution of noise is usually assigned as asserting or hypothesizing a statement of fact. The one encountered the most is Gaussian noise, e.g. the Blackman-Tukey windowsmoothing procedure is associated with the hypothesis that the data are a realization of a stationary Gaussian random process [6]. Of course there is nothing to prevent one from applying a procedure to any set of data no matter whether the hypothesis holds or not. Moreover, using a different type of noise may have no serious impact when one wants merely to get a qualitative feature from the data studied. However, use of the wrong noise model is definitely not acceptable in forward methods for deconvolution when the potential function contains noise-related parameter(s). Assigning a noise distribution different from real situation can be very costly, as pointed out by Tukey et al. [11].

There remain further investigations to be done for the noise in dynamic SIMS profiles. Changing the matrix composition may have effect on the MSE and mean relation. It will also be interesting to see whether such a Poissonian relation still holds when the experimental condition is further changed, such as using another element from a different type of source as the primary ion. 


\section{ACKNOWLEDGEMENT}

This work is supported by the EPSRC funding under the grant GR/K32715. 
[1] M. G. Dowsett and R. D. Barlow, Anal. Chim. Acta 297, 253 (1994).

[2] M. G. Dowsett, G. Rowlands, P. N. Allen, and R. D. Barlow, Surf. Interface Anal. 21, 310 (1994).

[3] M. G. Dowsett, R. D. Barlow, and P. N. Allen, J. Vac. Sci. Technol. B 12, 186 (1994).

[4] G. A. Cooke, M. G. Dowsett, P. N. Allen, R. Collins, and K. Miethe, J. Vac. Sci. Technol. B 14, $132(1996)$.

[5] S. O. Rice, "Mathematical Analysis of Random Noise" in Selected Papers on Noise and Stochastic Processes, edited by N. Wax, Dover Publications, Inc., New York, 1954, p. 133.

[6] E. T. Jaynes, "Bayesian Spectrum and Chirp Analysis" in Maximum-Entropy and Bayesian Spectral Analysis and Estimation Problems, edited by C. R. Smith and G. J. Erickson, D. Reidel Publishing Company, Dordrecht, Holland, 1987, p. 1.

[7] L. Cohen, Time-frequency Analysis, Prentice Hall PTR, New Jersey, 1995.

[8] G. M. Viswanathan, V. Afanasyev, S. V. Buldyrev, E. J. Murphy, P. A. Prince, and H. E. Stanley, Nature 381, 413 (1996).

[9] See A. Benninghoven, F. G. Rüdenauer, and H. W. Werner, Secondary Ion Mass Spectrometry, John Wiley and Sons, New York, 1987, p. 1056 and the references thereafter.

[10] J. B. Clegg, A. E. Morgan, H. A. M. de Grefte, F. Simondet, A. Huber, G. Blackmore, M. G. Dowsett, D. E. Sykes, C. W. Magee, and V. R. Deline, Surf. Interface Anal. 6, 162 (1984). 
[11] J. W. Tukey, P. Bloomfield, D. Brillinger, and W. S. Cleveland, "The Practice of Spectrum Analysis", notes on a course given in Princeton, N. J., in December, 1980. 


\section{TABLES}

TABLE I. Settings for the B staircase samples

\begin{tabular}{|c|c|c|}
\hline Sample/Run No. & Primary Beam & Primary Beam \\
\hline & Energy $E_{p}(\mathrm{keV})$ & Current $I_{p}(\mathrm{nA})$ \\
\hline $14.01 / 1.1$ & 0.5 & 243 \\
\hline $14.01 / 1.2$ & 2.0 & 447 \\
\hline $17.03 / 1.2$ & 0.5 & 243 \\
\hline $17.03 / 1.3$ & 2.0 & 447 \\
\hline $17.03 / 1.4$ & 2.0 & 390 \\
\hline $25.01 / 1.1$ & 0.5 & 243 \\
\hline $25.01 / 1.2$ & 2.0 & 447 \\
\hline $39.02 / 1.1$ & 2.0 & 257 \\
\hline $39.02 / 1.2$ & 2.0 & 469 \\
\hline $39.02 / 1.4$ & 0.5 & 310 \\
\hline $39.02 / 1.4 \mathrm{a}$ & 0.5 & 310 \\
\hline $39.02 / 1.4 \mathrm{~b}$ & 0.5 & 310 \\
\hline
\end{tabular}


TABLE II. Settings for the Si only profiles

\begin{tabular}{|c|c|c|c|c|}
\hline \multirow[t]{2}{*}{ Sample/Run No. } & \multicolumn{3}{|c|}{ Primary Beam } & Primary Beam \\
\hline & \multicolumn{3}{|c|}{ Energy $E_{p}(\mathrm{keV})$} & Current $I_{p}(\mathrm{nA})$ \\
\hline $14.01 / 2.1$ & \multicolumn{3}{|c|}{0.5} & $0.014-133$ \\
\hline $14.01 / 2.3$ & \multicolumn{3}{|c|}{2.0} & $0.0095-120$ \\
\hline $14.01 / 2.4$ & \multicolumn{3}{|c|}{0.5} & $0.0064-260$ \\
\hline $14.01 / 2.5$ & \multicolumn{3}{|c|}{0.5} & $0.10-180$ \\
\hline $14.01 / 2.6$ & \multicolumn{3}{|c|}{0.5} & $0.01-160$ \\
\hline \multirow[t]{2}{*}{ Sample/Run No. } & \multirow{2}{*}{\multicolumn{2}{|c|}{$\begin{array}{c}\text { Frame Time } \\
t_{f}(\mathrm{sec})\end{array}$}} & Areal & Sample Stage \\
\hline & & & (\% crater) & $\operatorname{Bias} V_{s}(\mathrm{~V})$ \\
\hline $14.01 / 2.1$ & \multicolumn{2}{|l|}{10} & 15 & 25.0 \\
\hline $14.01 / 2.3$ & \multicolumn{2}{|l|}{1} & 15 & 20.0 \\
\hline $14.01 / 2.4$ & \multicolumn{2}{|l|}{1} & 15 & 25.0 \\
\hline $14.01 / 2.5$ & \multicolumn{2}{|l|}{1} & 100 & 25.0 \\
\hline $14.01 / 2.6$ & \multicolumn{2}{|l|}{1} & 100 & 25.0 \\
\hline
\end{tabular}




\section{FIGURE CAPTIONS}

FIG. 1. A typical SIMS profile for a boron staircases in silicon (Sample 39.02, Run No: 1.1), obtained using $2.0 \mathrm{keV}{ }^{16} \mathrm{O}_{2}^{+}$ion beam, current $260 \mathrm{nA}$. The four steps in the boron channel between frames 200 and 3500 correspond to dopant levels of $3 \times 10^{16}, 1 \times 10^{17}, 1 \times 10^{18}$ and $1 \times 10^{19}$ atoms $/ \mathrm{cm}^{3}$ respectively.

FIG. 2. $\sigma$ vs $s_{m}$ for the noise in both boron and silicon mass channels of the staircase samples. The dashed and dotted lines are for the Poissonian and Gaussian noise respectively.

FIG. 3. $\sigma$ vs $s_{m}$ for the noise in the silicon only profiles. The dashed and dotted lines are corresponding to the relations of Poissonian and Gaussian noise respectively.

FIG. 4. The counts of channeltron vs $I_{p}$. The $I_{p}$ in (a) was increased monotonically, while in (b) it was raised and reduced as shown by the arrows. The lines connecting the data points are drawn to guide eyes. 


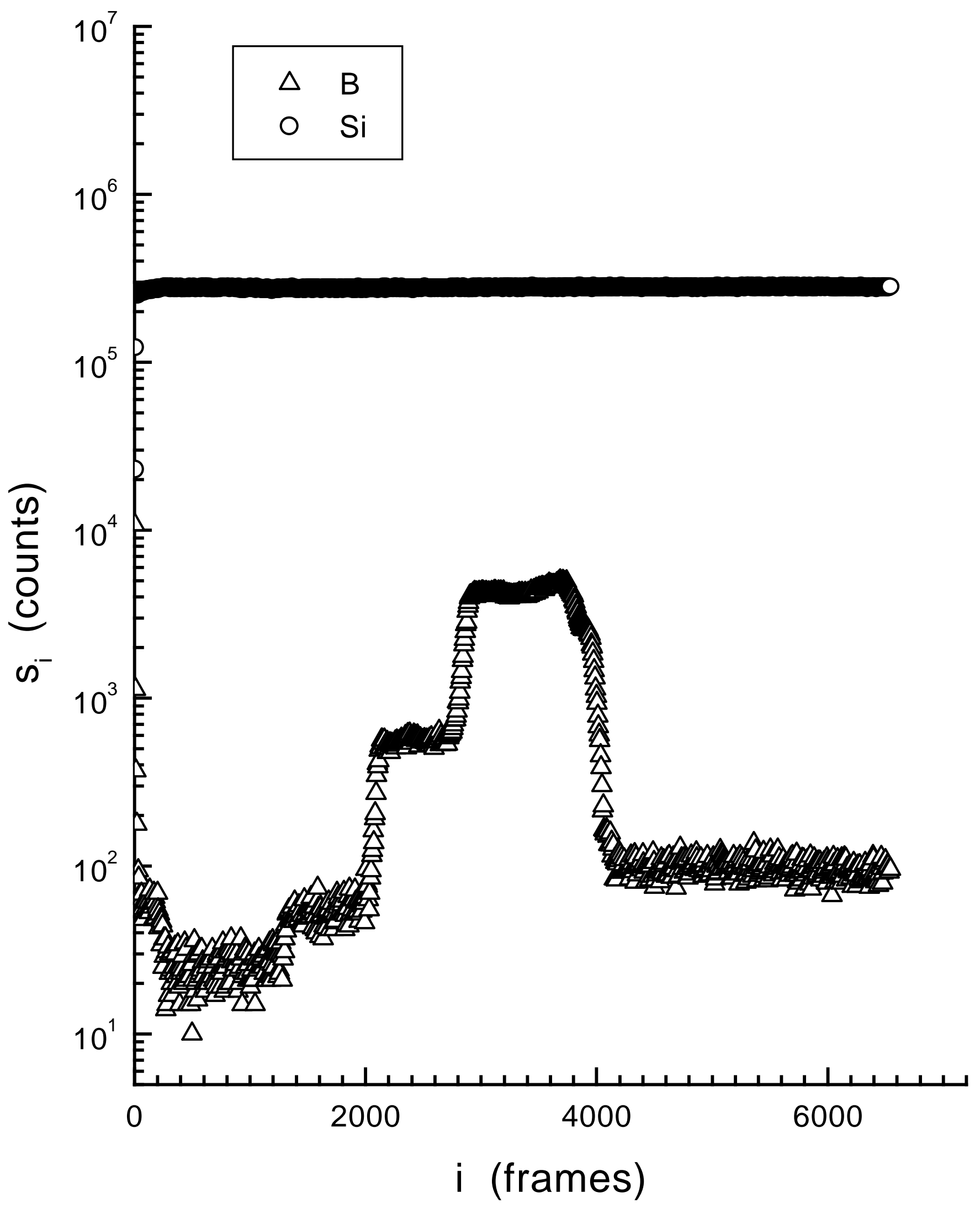




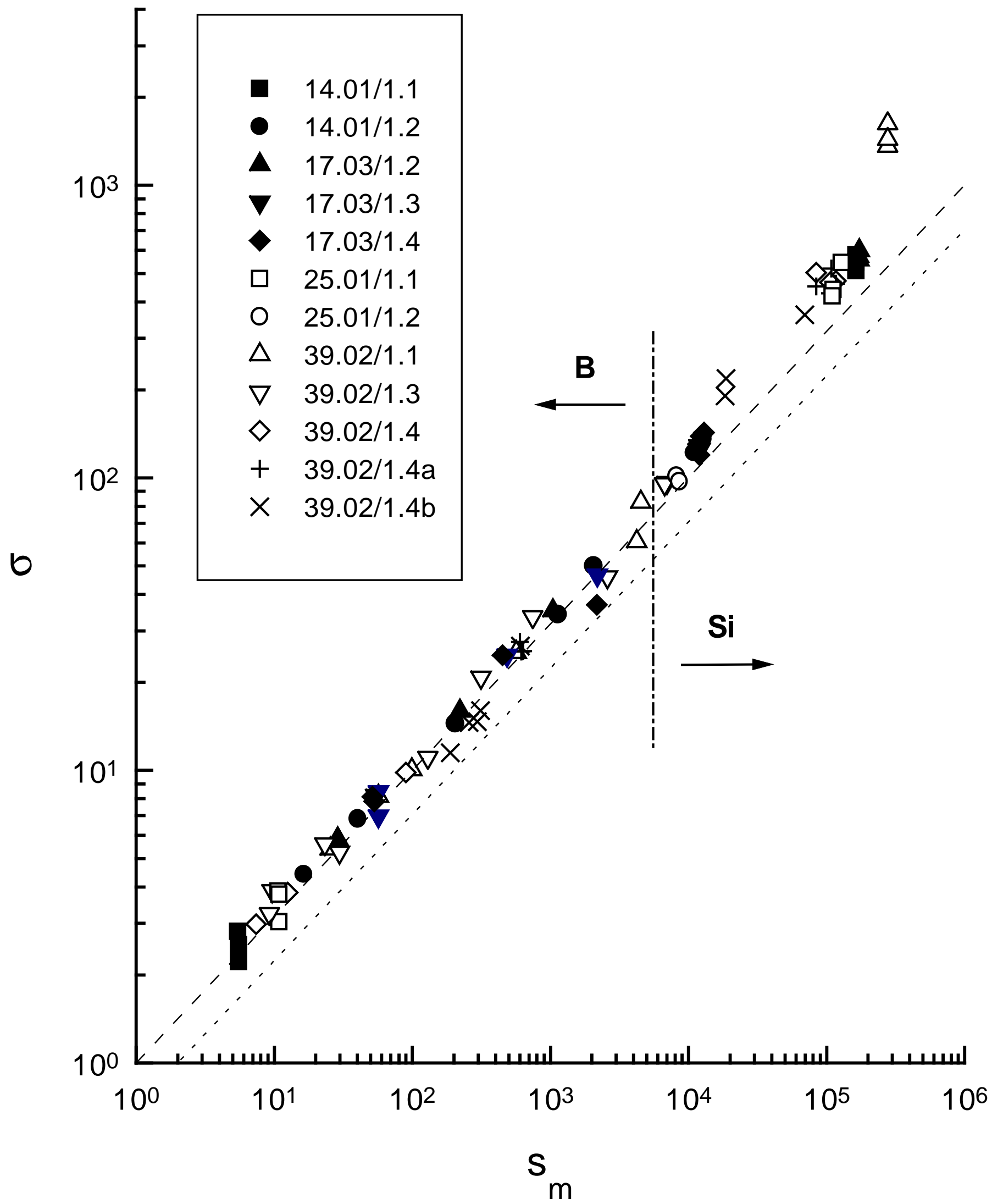




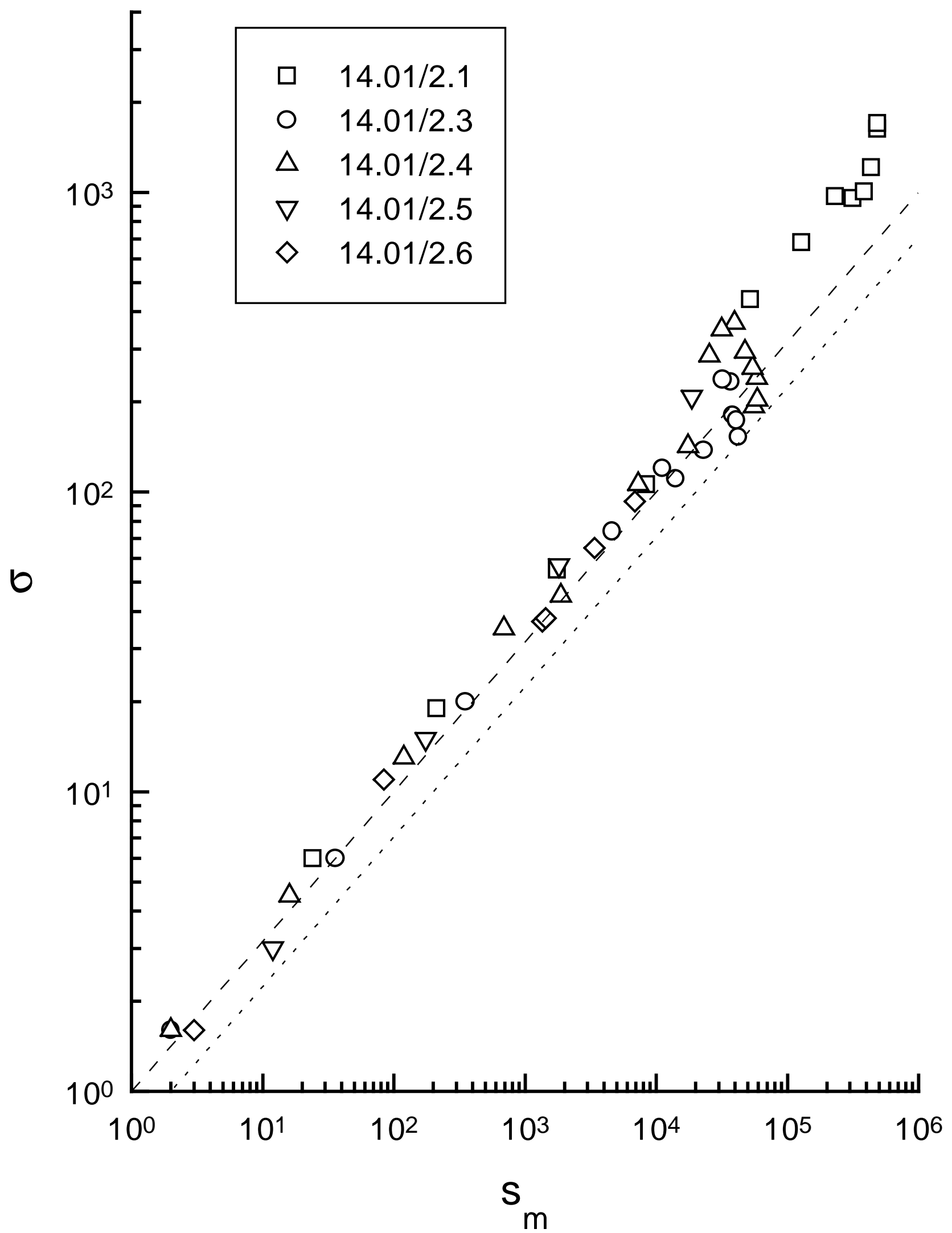






